\title{
Chiral density wave versus pion condensation at finite density and zero temperature
}

\author{
Jens O. Andersen ${ }^{1,2, *}$ and Patrick Kneschke ${ }^{3, \dagger}$ \\ ${ }^{1}$ Department of Physics, Faculty of Natural Sciences, NTNU, Norwegian University of Science and Technology, \\ Hogskoleringen 5, N-7491 Trondheim, Norway \\ ${ }^{2}$ Niels Bohr International Academy, Blegdamsvej 17, Copenhagen 2100, Denmark \\ ${ }^{3}$ Faculty of Science and Technology, University of Stavanger, N-4036 Stavanger, Norway
}

(Received 9 February 2018; published 11 April 2018)

\begin{abstract}
The quark-meson model is often used as a low-energy effective model for QCD to study the chiral transition at finite temperature $T$, baryon chemical potential $\mu_{B}$, and isospin chemical potential $\mu_{I}$. We determine the parameters of the model by matching the meson and quark masses, as well as the pion decay constant to their physical values using the on shell (OS) and modified minimal subtraction $(\overline{\mathrm{MS}})$ schemes. In this paper, the existence of different phases at zero temperature is studied. In particular, we investigate the competition between an inhomogeneous chiral condensate and a homogeneous pion condensate. For the inhomogeneity, we use a chiral-density wave ansatz. For a sigma mass of $600 \mathrm{MeV}$, we find that an inhomogeneous chiral condensate exists only for pion masses below approximately $37 \mathrm{MeV}$. We also show that due to our parameter fixing, the onset of pion condensation takes place exactly at $\mu_{I}^{c}=\frac{1}{2} m_{\pi}$ in accordance with exact results.
\end{abstract}

DOI: 10.1103/PhysRevD.97.076005

\section{INTRODUCTION}

The phases of dense QCD as functions of the baryon chemical potential $\mu_{B}$ or the quark chemical potential $\mu=$ $\frac{1}{3} \mu_{B}$ have been studied in detail since the first phase diagram was suggested in the 1970s [1-3]. For baryon chemical potentials lower than the nucleon mass $m_{N}$ and at $T=0$, we are in the vacuum phase. For larger values of the baryon chemical potential, one expected a first-order transition to quark matter, a phase in which chiral symmetry is approximately restored and where quarks are no longer confined to the individual nucleons. It turns out that the phase diagram is very rich. For asymptotically large values of the quark chemical potential, we have one of the few rigorous results. Due to asymptotic freedom, the system is weakly interacting and an attractive quark-quark interaction from one-gluon exchange gives rise to color superconductivity and the so-called color-flavor locked phase. For lower values of the quark chemical potential, one cannot use perturbative QCD, and instead one has to use low-energy effective models of QCD such as the quarkmeson (QM) model or Nambu-Jona-Lasinio (NJL) model.

\footnotetext{
*andersen@tf.phys.ntnu.no

patrick.kneschke@uis.no
}

Published by the American Physical Society under the terms of the Creative Commons Attribution 4.0 International license. Further distribution of this work must maintain attribution to the author(s) and the published article's title, journal citation, and DOI. Funded by SCOAP.
These models predict a plethora of superconducting phases depending on e.g. the quark masses and other external parameters such as magnetic fields [4-6]. Other possibilities include the quarkyonic phase $[7,8]$, whose existence is unambiguously argued in the large- $N_{c}$ limit, where $N_{c}$ is the number of colors.

Another interesting aspect of the QCD phase diagram at $T=0$ is the possibility of inhomogeneous phases, see $[9,10]$ for reviews. These are phases where two quarks (or a quark and an antiquark) with momenta $\mathbf{p}+\mathbf{q}$ and $-\mathbf{p}+\mathbf{q}$ pair, resulting in a Cooper pair with a net momentum $2 \mathbf{q}$ and a complex order parameter of the form $\Delta e^{2 i \mathbf{q} \cdot \mathbf{x}}$.

Instead of considering only the baryon chemical potential, one can allow for an independent chemical potential $\mu_{f}$ for each quark flavor $f$. For two quark flavors, using $\mu_{u}$ and $\mu_{d}$ is equivalent to using $\mu_{B}$ and an isospin chemical potential $\mu_{I}$. Systems at zero baryon chemical potential and finite isospin chemical potential are of particular interest, since the fermion determinant is real and one can perform lattice simulations using standard importance sampling techniques, see e.g. [11-15]. The picture that emerges from the simulations is that at $T=0$, the chiral condensate is constant for $\mu_{I}$ below a critical value, $\mu_{I}^{c}=\frac{1}{2} m_{\pi} \cdot{ }^{1}$ The critical value of the isospin chemical potential marks the onset of pion condensation, and the transition is of second order. This picture is consistent with the predictions of chiral perturbation theory $[16,17]$.

\footnotetext{
${ }^{1}$ Depending on convention, $\mu_{I}^{c}=m_{\pi}$ is also frequently found in the literature.
} 
In addition to chiral perturbation theory [16-20], there have also been a number of other approaches and model calculations studying various aspects of the QCD phase diagram at finite isospin density. These include the resonance gas model [21], random matrix models [22], the NJL model [23-34], the quark-meson model [35-37], perturbative QCD [38], and hard-thermal-loop perturbation theory [39].

In the present paper, we use the quark-meson model to study possible phases at $T=0$ and at finite baryon and isospin chemical potentials. In particular, we extend certain aspects of earlier studies [40-43] by looking at the competition between an inhomogeneous chiral condensate and a homogeneous pion condensate. Studies of the competition between homogeneous and inhomogeneous condensates have been carried out in the $1+1$ dimensional NJL model in [44-46].

The paper is organized as follows. In Sec. II, we briefly discuss the quark-meson model, and in Sec. III we calculate the effective potential in the mean-field approximation. In Sec. IV, we present the phase diagram in the $\mu-\mu_{I}$ plane at zero temperature, and in Sec. V, we summarize and conclude. In Appendix A, we list a number of integrals needed in the calculations, while Appendix B provides the reader with some details of how the parameters are determined. In Appendix $\mathrm{C}$, we show that the critical isospin chemical potential is exactly $\mu_{I}^{c}=\frac{1}{2} m_{\pi}$ in our approximation.

\section{QUARK-MESON MODEL}

The Lagrangian of the two-flavor quark-meson model in Minkowski space is

$$
\begin{aligned}
\mathcal{L}= & \frac{1}{2}\left[\left(\partial_{\mu} \sigma\right)\left(\partial^{\mu} \sigma\right)+\left(\partial_{\mu} \pi_{3}\right)\left(\partial^{\mu} \pi_{3}\right)\right] \\
& +\left(\partial_{\mu}+2 i \mu_{I} \delta_{\mu}^{0}\right) \pi^{+}\left(\partial^{\mu}-2 i \mu_{I} \delta_{0}^{\mu}\right) \pi^{-} \\
& -\frac{1}{2} m^{2}\left(\sigma^{2}+\pi_{3}^{2}+2 \pi^{+} \pi^{-}\right)-\frac{\lambda}{24}\left(\sigma^{2}+\pi_{3}^{2}+2 \pi^{+} \pi^{-}\right)^{2} \\
& +h \sigma+\bar{\psi}\left[i \gamma+\mu_{f} \gamma^{0}-g\left(\sigma+i \gamma^{5} \tau \cdot \pi\right)\right] \psi
\end{aligned}
$$

where $\psi$ is a color $N_{c}$-plet, a four-component Dirac spinor as well as a flavor doublet

$$
\psi=\left(\begin{array}{l}
u \\
d
\end{array}\right)
$$

and $\mu_{f}=\operatorname{diag}\left(\mu_{u}, \mu_{d}\right)$, where $\mu_{u}$ and $\mu_{d}$, are the quark chemical potentials, $\mu_{I}$ is the isospin chemical potential, $\tau_{i}$ $(i=1,2,3)$ are the Pauli matrices in flavor space, $\boldsymbol{\pi}=\left(\pi_{1}, \pi_{2}, \pi_{3}\right)$, and $\pi^{ \pm}=\frac{1}{\sqrt{2}}\left(\pi_{1} \pm i \pi_{2}\right)$.

Apart from the global $S U\left(N_{c}\right)$ symmetry, the Lagrangian (1) has a $U(1)_{B} \times S U(2)_{L} \times S U(2)_{R}$ symmetry for $h=0$ and a $U(1)_{B} \times S U(2)_{V}$ symmetry for $h \neq 0$. When $\mu_{u} \neq \mu_{d}$, this symmetry is reduced to $U(1)_{B} \times U_{I_{3} L}(1) \times$ $U_{I_{3} R}(1)$ for $h=0$ and $U(1)_{B} \times U_{I_{3}}(1)$ for $h \neq 0$.
The number density associated with a chemical potential $\mu_{A}$ is

$$
n_{A}=-\frac{\partial V}{\partial \mu_{A}},
$$

where $V$ is the effective potential. The baryon and isospin densities can be expressed in terms of the quark densities $n_{u}$ and $n_{d}$ as

$$
\begin{aligned}
n_{B} & =\frac{1}{3}\left(n_{u}+n_{d}\right), \\
n_{I} & =n_{u}-n_{d} .
\end{aligned}
$$

Equations (4)-(5) together with the chain rule can be used to derive relations among the baryon and isospin chemical potentials and the quark chemical potentials. We have

$$
\begin{aligned}
n_{I} & =-\frac{\partial V}{\partial \mu_{I}} \\
& =-\left(\frac{\partial V}{\partial \mu_{u}}-\frac{\partial V}{\partial \mu_{d}}\right) \\
& =-\left(\frac{\partial \mu_{u}}{\partial \mu_{I}} \frac{\partial V}{\partial \mu_{u}}+\frac{\partial \mu_{d}}{\partial \mu_{I}} \frac{\partial V}{\partial \mu_{d}}\right) .
\end{aligned}
$$

This yields

$$
\frac{\partial \mu_{u}}{\partial \mu_{I}}=-\frac{\partial \mu_{u}}{\partial \mu_{I}}=1 .
$$

Similarly, we find $\frac{\partial \mu_{u}}{\partial \mu_{B}}=\frac{\partial \mu_{d}}{\partial \mu_{B}}=\frac{1}{3}$. From this, we find the following relations among the chemical potentials:

$$
\begin{aligned}
& \mu_{u}=\frac{1}{3} \mu_{B}+\mu_{I}, \\
& \mu_{d}=\frac{1}{3} \mu_{B}-\mu_{I} .
\end{aligned}
$$

Introducing the quark chemical potential $\mu=\frac{1}{3} \mu_{B}$ and inverting the relations (8)-(9), we find

$$
\begin{aligned}
\mu & =\frac{1}{2}\left(\mu_{u}+\mu_{d}\right), \\
\mu_{I} & =\frac{1}{2}\left(\mu_{u}-\mu_{d}\right) .
\end{aligned}
$$

In the following, we will express the equations and our result in terms of $\mu$ and $\mu_{I}$ instead of $\mu_{u}$ and $\mu_{d}$.

\section{EFFECTIVE POTENTIAL}

In the following, we allow for an inhomogeneous chiral condensate. To be specific, we consider a one-dimensional chiral-density wave with a wave vector $\mathbf{q}$ pointing in the positive $z$-direction. The expectation values of the fields are written as

$$
\begin{aligned}
\sigma & =\phi_{0} \cos (q z), \quad \pi_{3}=\phi_{0} \sin (q z) \\
\pi_{1} & =\pi_{0}, \quad \pi_{2}=0,
\end{aligned}
$$


where $\phi_{0}$ and $\pi_{0}$ are constant in space. The latter represents a homogeneous pion condensate. A pion condensate breaks the $U_{I_{3} L}(1) \times U_{I_{3} R}(1)$ symmetry to $U_{I_{3} V}(1)$ or the $U_{I_{3}}(1)$ symmetry. Introducing $\Delta=g \phi_{0}$ and $\rho=g \pi_{0}$, the tree-level potential in Euclidean space can be written as

$$
\begin{aligned}
V_{0}= & \frac{1}{2} \frac{q^{2}}{g^{2}} \Delta^{2}+\frac{1}{2} \frac{m^{2}}{g^{2}} \Delta^{2}+\frac{1}{2} \frac{m^{2}-4 \mu_{I}^{2}}{g^{2}} \rho^{2} \\
& +\frac{\lambda}{24 g^{4}}\left(\Delta^{2}+\rho^{2}\right)^{2}-\frac{h}{g} \Delta \cos (q z) \delta_{q, 0} .
\end{aligned}
$$

The Kronecker-delta $\delta_{q, 0}$ in the last term in Eq. (14) is necessary since a term $\Delta \cos (q z)$ vanishes for a nonzero $q$ upon integrating the free energy density over a sufficiently large spatial volume.

Expressing the parameters in the Lagrangian in terms of the sigma mass $m_{\sigma}$, pion mass $m_{\pi}$, pion decay constant $f_{\pi}$, and quark mass $m_{q}$, we find

$$
\begin{aligned}
& m^{2}=-\frac{1}{2}\left(m_{\sigma}^{2}-3 m_{\pi}^{2}\right), \quad \lambda=3 \frac{\left(m_{\sigma}^{2}-m_{\pi}^{2}\right)}{f_{\pi}^{2}}, \\
& g^{2}=\frac{m_{q}^{2}}{f_{\pi}^{2}}, \quad h=m_{\pi}^{2} f_{\pi} .
\end{aligned}
$$

Inserting these relations, we can write the tree-level potential as

$$
\begin{aligned}
V_{0}= & \frac{1}{2} f_{\pi}^{2} q^{2} \frac{\Delta^{2}}{m_{q}^{2}}-\frac{1}{4} f_{\pi}^{2}\left(m_{\sigma}^{2}-3 m_{\pi}^{2}\right) \frac{\Delta^{2}+\rho^{2}}{m_{q}^{2}}-2 \mu_{I}^{2} f_{\pi}^{2} \frac{\rho^{2}}{m_{q}^{2}} \\
& +\frac{1}{8} f_{\pi}^{2}\left(m_{\sigma}^{2}-m_{\pi}^{2}\right) \frac{\left(\Delta^{2}+\rho^{2}\right)^{2}}{m_{q}^{4}} \\
& -m_{\pi}^{2} f_{\pi}^{2} \frac{\Delta}{m_{q}} \cos (q z) \delta_{q, 0}
\end{aligned}
$$

Since we want to integrate out the fermions, we need the part of the Lagrangian that is bilinear in the quark fields

$$
\mathcal{L}_{2}=\bar{\psi}\left[i \gamma^{\mu} \partial_{\mu}+\left(\mu+\tau_{3} \mu_{I}\right) \gamma^{0}-\Delta e^{i \gamma^{5} \tau_{3} q z}-i \tau_{1} \gamma^{5} \rho\right] \psi .
$$

We next redefine the quark fields, $\psi \rightarrow e^{-\frac{1}{2} i \gamma^{5} \tau_{3} q x} \psi$ and $\bar{\psi} \rightarrow \bar{\psi} e^{-\frac{1}{2} i \gamma^{5} \tau_{3} q x}$. The transformation of the field $\psi$ amounts to a unitary transformation of the Dirac Hamiltonian, $\mathcal{H} \rightarrow \mathcal{H}^{\prime}=e^{\frac{1}{2} \gamma^{5} \tau_{3} q x} \mathcal{H} e^{-\frac{1}{2} \gamma^{5} \tau_{3} q x}$. The Dirac operator $D$ then reads

$$
D=\left[i \gamma^{\mu} \partial_{\mu}+\left(\mu+\tau_{3} \mu_{I}\right) \gamma^{0}-\Delta+\frac{1}{2} \gamma^{5} \gamma^{3} \tau_{3} q-i \tau_{1} \gamma^{5} \rho\right]
$$

In momentum space, the Dirac operator is

$D=\left[\not p+\left(\mu+\tau_{3} \mu_{I}\right) \gamma^{0}-\Delta+\frac{1}{2} \gamma^{5} \gamma^{3} \tau_{3} q-i \tau_{1} \gamma^{5} \rho\right]$.

The quark energies can be read off from the zeros of the determinant of the Dirac operator, which are found to be, see e.g. [47]

$$
\begin{aligned}
& E_{u}^{ \pm}=E\left( \pm q,-\mu_{I}\right), \quad E_{d}^{ \pm}=E\left( \pm q, \mu_{I}\right), \\
& E_{\bar{u}}^{ \pm}=E\left( \pm q, \mu_{I}\right), \quad E_{\bar{d}}^{ \pm}=E\left( \pm q,-\mu_{I}\right),
\end{aligned}
$$

where we have defined

$$
E\left(q, \mu_{I}\right)=\left[\left(\sqrt{p_{\perp}^{2}+\left(\sqrt{p_{\|}^{2}+\Delta^{2}}+\frac{q}{2}\right)^{2}}+\mu_{I}\right)^{2}+\rho^{2}\right]^{\frac{1}{2}},
$$

and where the wave vector $\mathbf{q}$ points in the positive $p_{\|}$ direction.

The one-loop contribution to the effective potential is

$$
V_{1}=-\frac{1}{2} N_{c} \int_{p}\left(E_{u}^{ \pm}+E_{d}^{ \pm}+E_{\bar{u}}^{ \pm}+E_{\bar{d}}^{ \pm}\right)
$$

where a sum over \pm is implied and the integral is in $d=$ $3-2 \epsilon$ dimensions (See Appendix A). The integral in Eq. (23) is ultraviolet divergent, and in order to isolate the divergences, we need to expand the energies in powers of $q$ and $\mu_{I}$ to the appropriate orders. This yields

$$
\begin{aligned}
V_{\mathrm{div}}= & -4 N_{c} \int_{p}\left[\sqrt{p^{2}+\Delta^{2}+\rho^{2}}+\frac{\mu_{I}^{2} \rho^{2}}{2\left(p^{2}+\Delta^{2}+\rho^{2}\right)^{\frac{3}{2}}}+\frac{3 q^{2} \mu_{I}^{2} \rho^{2}\left(4 \Delta^{2}+4 p_{\|}^{2}-p_{\perp}^{2}-\rho^{2}\right)}{16\left(p^{2}+\Delta^{2}+\rho^{2}\right)^{\frac{7}{2}}}\right. \\
& \left.+\frac{q^{2}\left(p_{\perp}^{2}+\rho^{2}\right)}{8\left(p^{2}+\Delta^{2}+\rho^{2}\right)^{\frac{3}{2}}}+\frac{q^{4}\left(p_{\perp}^{2}+\rho^{2}\right)\left(4 \Delta^{2}+4 p_{\|}^{2}-p_{\perp}^{2}-\rho^{2}\right)}{128\left(p^{2}+\Delta^{2}+\rho^{2}\right)^{\frac{7}{2}}}\right] \\
= & \frac{2 N_{c}}{(4 \pi)^{2}}\left(\frac{e^{\gamma_{E}} \Lambda^{2}}{\Delta^{2}+\rho^{2}}\right)^{\epsilon}\left\{2\left(\Delta^{2}+\rho^{2}\right)^{2} \Gamma(-2+\epsilon)+q^{2} \Delta^{2} \Gamma(\epsilon)-4 \mu_{I}^{2} \rho^{2} \Gamma(\epsilon)-2 q^{2} \mu_{I}^{2} \frac{\Delta^{2} \rho^{2}}{\left(\Delta^{2}+\rho^{2}\right)^{2}} \Gamma(2+\epsilon)\right. \\
& \left.-\frac{q^{4}}{12} \frac{\Delta^{2}}{\left(\Delta^{2}+\rho^{2}\right)^{2}}\left[(1-\epsilon) \Delta^{2}+2 \rho^{2}\right] \Gamma(1+\epsilon)\right\} .
\end{aligned}
$$


The remainder $V_{\text {fin }}$ is finite and reads

$$
V_{\text {fin }}=V_{1}-V_{\text {div }} \text {. }
$$

Note that $V_{\text {fin }}$ can be evaluated directly in $d=3$ dimensions. In the case $\rho=\mu_{I}=0$, one can calculate $V_{\text {fin }}$ analytically, see Ref. [48] for an explicit evaluation. In the present case, it must be evaluated numerically. Using the expressions for the integrals listed in Appendix A, we can write the unrenormalized one-loop effective potential $V=V_{0}+V_{1}$ as

$$
\begin{aligned}
V= & \frac{1}{2} \frac{q^{2}}{g^{2}} \Delta^{2}+\frac{1}{2} \frac{m^{2}}{g^{2}} \Delta^{2}+\frac{1}{2} \frac{m^{2}-4 \mu_{I}^{2}}{g^{2}} \rho^{2}+\frac{\lambda}{24 g^{4}}\left(\Delta^{2}+\rho^{2}\right)^{2}-\frac{h}{g} \Delta \cos (q z) \delta_{q, 0} \\
& +\frac{2 N_{c}}{(4 \pi)^{2}}\left(\frac{\Lambda^{2}}{\Delta^{2}+\rho^{2}}\right)^{\epsilon}\left[\left(\Delta^{2}+\rho^{2}\right)^{2}\left(\frac{1}{\epsilon}+\frac{3}{2}\right)+q^{2} \Delta^{2} \frac{1}{\epsilon}-4 \mu_{I}^{2} \rho^{2} \frac{1}{\epsilon}-\frac{q^{4}}{12} \frac{\Delta^{2}\left(\Delta^{2}+2 \rho^{2}\right)}{\left(\Delta^{2}+\rho^{2}\right)^{2}}-2 q^{2} \mu_{I}^{2} \frac{\Delta^{2} \rho^{2}}{\left(\Delta^{2}+\rho^{2}\right)^{2}}\right] \\
& +V_{\text {fin }}+\mathcal{O}(\epsilon) .
\end{aligned}
$$

The unrenormalized one-loop effective potential contains poles in $\epsilon$, which are removed by mass and coupling constant renormalization. In the $\overline{\mathrm{MS}}$ scheme this is achieved by making the substitutions $m^{2} \rightarrow Z_{m^{2}} m^{2}, \lambda \rightarrow Z_{\lambda} \lambda, g^{2} \rightarrow Z_{g^{2}} g^{2}$, and $h \rightarrow Z_{h} h$, where

$$
Z_{m^{2}}=1+\frac{4 N_{c} g^{2}}{(4 \pi)^{2} \epsilon}, \quad Z_{\lambda}=1+\frac{8 N_{c}}{(4 \pi)^{2} \epsilon}\left[g^{2}-6 \frac{g^{4}}{\lambda}\right], \quad Z_{g^{2}}=1+\frac{4 N_{c} g^{2}}{(4 \pi)^{2} \epsilon}, \quad Z_{h}=1+\frac{2 N_{c} g^{2}}{(4 \pi)^{2} \epsilon} .
$$

The renormalized one-loop effective potential then reads

$$
\begin{aligned}
V_{1-\text { loop }}= & \frac{1}{2} \frac{q^{2}}{g_{\overline{\mathrm{MS}}}^{2}} \Delta^{2}+\frac{1}{2} \frac{m_{\overline{\mathrm{MS}}}^{2}}{g_{\overline{\mathrm{MS}}}^{2}} \Delta^{2}+\frac{1}{2} \frac{m_{\overline{\mathrm{MS}}}^{2}-4 \mu_{I}^{2}}{g_{\overline{\mathrm{MS}}}^{2}} \rho^{2}+\frac{\lambda_{\overline{\mathrm{MS}}}}{24 g_{\overline{\mathrm{MS}}}^{4}}\left(\Delta^{2}+\rho^{2}\right)^{2}-\frac{h_{\overline{\mathrm{MS}}}}{g_{\overline{\mathrm{MS}}}} \Delta \cos (q z) \delta_{q, 0} \\
& +\frac{2 N_{c}}{(4 \pi)^{2}}\left\{\left[\left(\Delta^{2}+\rho^{2}\right)^{2}+q^{2} \Delta^{2}-4 \mu_{I}^{2} \rho^{2}\right] \log \left(\frac{\Lambda^{2}}{\Delta^{2}+\rho^{2}}\right)+\frac{3}{2}\left(\Delta^{2}+\rho^{2}\right)^{2}-\frac{q^{4}}{12} \frac{\Delta^{2}\left(\Delta^{2}+2 \rho^{2}\right)}{\left(\Delta^{2}+\rho^{2}\right)^{2}}-2 q^{2} \mu_{I}^{2} \frac{\Delta^{2} \rho^{2}}{\left(\Delta^{2}+\rho^{2}\right)^{2}}\right\}+V_{\text {fin }},
\end{aligned}
$$

where the subscript $\overline{\mathrm{MS}}$ indicates that the parameters are running with the renormalization scale $\Lambda$. In Appendix B, we discuss how one can express the parameters in the $\overline{\mathrm{MS}}$ scheme in terms of physical masses and couplings. Using Eqs. (B14)-(B17), the final expression for the one-loop effective potential in the large- $N_{c}$ limit becomes

$$
\begin{aligned}
V_{1-\text { loop }}= & \frac{1}{2} f_{\pi}^{2} q^{2}\left\{1-\frac{4 m_{q}^{2} N_{c}}{(4 \pi)^{2} f_{\pi}^{2}}\left[\log \frac{\Delta^{2}+\rho^{2}}{m_{q}^{2}}+F\left(m_{\pi}^{2}\right)+m_{\pi}^{2} F^{\prime}\left(m_{\pi}^{2}\right)\right]\right\} \frac{\Delta^{2}}{m_{q}^{2}} \\
& +\frac{3}{4} m_{\pi}^{2} f_{\pi}^{2}\left\{1-\frac{4 m_{q}^{2} N_{c}}{(4 \pi)^{2} f_{\pi}^{2}} m_{\pi}^{2} F^{\prime}\left(m_{\pi}^{2}\right)\right\} \frac{\Delta^{2}+\rho^{2}}{m_{q}^{2}} \\
& -\frac{1}{4} m_{\sigma}^{2} f_{\pi}^{2}\left\{1+\frac{4 m_{q}^{2} N_{c}}{(4 \pi)^{2} f_{\pi}^{2}}\left[\left(1-\frac{4 m_{q}^{2}}{m_{\sigma}^{2}}\right) F\left(m_{\sigma}^{2}\right)+\frac{4 m_{q}^{2}}{m_{\sigma}^{2}}-F\left(m_{\pi}^{2}\right)-m_{\pi}^{2} F^{\prime}\left(m_{\pi}^{2}\right)\right]\right\} \frac{\Delta^{2}+\rho^{2}}{m_{q}^{2}} \\
& -2 \mu_{I}^{2} f_{\pi}^{2}\left\{1-\frac{4 m_{q}^{2} N_{c}}{(4 \pi)^{2} f_{\pi}^{2}}\left[\log \frac{\Delta^{2}+\rho^{2}}{m_{q}^{2}}+F\left(m_{\pi}^{2}\right)+m_{\pi}^{2} F^{\prime}\left(m_{\pi}^{2}\right)\right]\right\} \frac{\rho^{2}}{m_{q}^{2}} \\
& +\frac{1}{8} m_{\sigma}^{2} f_{\pi}^{2}\left\{1-\frac{4 m_{q}^{2} N_{c}}{(4 \pi)^{2} f_{\pi}^{2}}\left[\frac{4 m_{q}^{2}}{m_{\sigma}^{2}}\left(\frac{\log \Delta^{2}+\rho^{2}}{m_{q}^{2}}-\frac{3}{2}\right)-\left(1-\frac{4 m_{q}^{2}}{m_{\sigma}^{2}}\right) F\left(m_{\sigma}^{2}\right)+F\left(m_{\pi}^{2}\right)+m_{\pi}^{2} F^{\prime}\left(m_{\pi}^{2}\right)\right]\right\} \frac{\left(\Delta^{2}+\rho^{2}\right)^{2}}{m_{q}^{4}} \\
& -\frac{1}{8} m_{\pi}^{2} f_{\pi}^{2}\left[1-\frac{4 m_{q}^{2} N_{c}}{(4 \pi)^{2} f_{\pi}^{2}} m_{\pi}^{2} F^{\prime}\left(m_{\pi}^{2}\right)\right] \frac{\left(\Delta^{2}+\rho^{2}\right)^{2}}{m_{q}^{4}}-m_{\pi}^{2} f_{\pi}^{2}\left[1-\frac{4 m_{q}^{2} N_{c}}{(4 \pi)^{2} f_{\pi}^{2}} m_{\pi}^{2} F^{\prime}\left(m_{\pi}^{2}\right)\right] \frac{\Delta}{m_{q}} \cos (q z) \delta_{q, 0} \\
& -\frac{N_{c}}{6(4 \pi)^{2}}\left[q^{4} \frac{\Delta^{2}\left(\Delta^{2}+2 \rho^{2}\right)}{\left(\Delta^{2}+\rho^{2}\right)^{2}}+24 q^{2} \mu_{I}^{2} \frac{\Delta^{2} \rho^{2}}{\left(\Delta^{2}+\rho^{2}\right)^{2}}\right]+V_{\text {fin }} .
\end{aligned}
$$


The effective potential must be independent of $q$ in the limit $\Delta \rightarrow 0$, it cannot depend on the wave vector if the magnitude of the order parameter is zero. We have checked numerically that this is the case for $V_{1-\text { loop }}$ in Eq. (29).

The matter part of the one-loop effective potential is

$$
\begin{aligned}
V_{\mathrm{mat}}= & -N_{c} T \int_{p}\left\{\log \left[1+e^{-\beta\left(E_{u}^{ \pm}-\mu\right)}\right]\right. \\
& +\log \left[1+e^{-\beta\left(E_{d}^{ \pm}-\mu\right)}\right] \\
& \left.+\log \left[1+e^{-\beta\left(E_{\bar{u}}^{ \pm}+\mu\right)}\right]+\log \left[1+e^{-\beta\left(E_{\bar{d}}^{ \pm}+\mu\right)}\right]\right\} .
\end{aligned}
$$

In the limit $T \rightarrow 0$, this contribution reads

$V_{\mathrm{mat}}=N_{c} \int_{p}\left[\left(E_{u}^{ \pm}-\mu\right) \theta\left(\mu-E_{u}^{ \pm}\right)+\left(E_{d}^{ \pm}-\mu\right) \theta\left(\mu-E_{d}^{ \pm}\right)\right]$.

The complete one-loop effective potential is then the sum of Eqs. (29) and (31) and is denoted by $V_{\text {full. }}$.

\section{PHASE DIAGRAM}

In this section, we discuss the phase diagram in the $\mu-\mu_{I}$ plane at $T=0$. In the numerical work below, we set $N_{c}=3, m_{\sigma}=600 \mathrm{MeV}$, and $f_{\pi}=93 \mathrm{MeV}$. In the chiral limit $m_{\pi}=0$, while at the physical point, we set $m_{\pi}=140 \mathrm{MeV}$.

\section{A. Homogeneous chiral condensate}

We first restrict ourselves to a homogeneous chiral condensate as well as a homogeneous pion condensate, i.e. we set $q=0$ in Eqs. (29) and (31). In the remainder of this section we consider only the physical point.

In Fig. 1, we show in the upper panel, the chiral condensate (blue line) and pion condensate (red line) as functions of the isospin chemical potential $\mu_{I}$ for $\mu=0$ at vanishing temperature. The onset of pion condensation is at $\mu_{I}=\frac{1}{2} m_{\pi}$. The pion condensate increases, while the chiral condensate decreases. One can view this as a rotation of the chiral condensate into a pion condensate as $\mu_{I}$ increases. For $\mu_{I}<\frac{1}{2} m_{\pi}$, the chiral condensate is constant, which reflects the Silver Blaze property of the vacuum phase: physical quantities are independent of the isospin chemical potential for $\mu_{I}<\mu_{I}^{c}$, where the critical chemical potential is $\mu_{I}^{c}=$ $\frac{1}{2} m_{\pi}$ [49]. In the lower panel of Fig. 1, we also show the chiral condensate (blue line) and pion condensate (red line) as functions of the isospin chemical potential $\mu_{I}$, now for $\mu=260 \mathrm{MeV}$. We notice that there is a region of $\mu$, where $\Delta$ decreases before the onset of pion condensation. This corresponds to a phase which is different from the vacuum phase since the thermodynamic observables (here the chiral condensate) depend on the isospin chemical potential.

More generally, the effective potential, and therefore thermodynamic observables are independent of $\mu$ and $\mu_{I}$ in
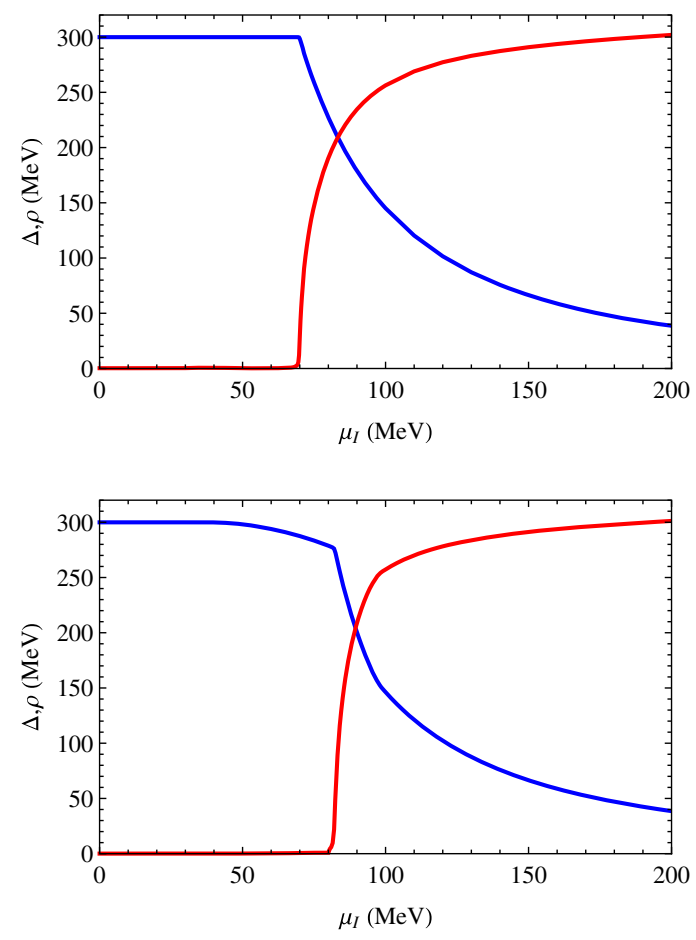

FIG. 1. Chiral condensate (blue line) and pion condensate (red line) as functions of the isospin chemical potential $\mu_{I}$ for $\mu=0$ (upper panel) and $\mu=260 \mathrm{MeV}$ (lower panel) at the physical point and $T=0$.

the region bounded by the $\mu_{I}$ and $\mu$ axes, and the straight lines given by $\mu+\mu_{I}=g f_{\pi}=m_{q}$ and $\mu_{I}=\mu_{i}^{c}=\frac{1}{2} m_{\pi}$. In particular, the quark and isospin densities, which are defined by

$$
n_{q}=-\frac{\partial V_{\text {full }}}{\partial \mu}, \quad n_{I}=-\frac{\partial V_{\text {full }}}{\partial \mu_{I}}
$$

vanish in this region. We therefore refer to this region as the vacuum phase. This is shown in Fig. 2, where the vacuum phase is bounded below the solid blue and red lines. The red line shows the phase boundary between a phase with $\rho=0$ and a pion-condensed phase. The transition is second order when the red line is solid and first order when it is dashed. The solid dot indicates the position of the critical end point where the first-order line ends. The critical end point is located at $\left(\mu, \mu_{I}\right)=(264,91) \mathrm{MeV}$. The green line indicates the boundary between a chirally broken phase and a phase where chiral symmetry is approximately restored. For a sigma mass of $m_{\sigma} \geq 600 \mathrm{MeV}$ this transition is a crossover before it attaches to the dashed red line. ${ }^{2}$ The region bounded by the three lines is a phase with chiral symmetry breaking but no pion condensate. The effective

\footnotetext{
${ }^{2}$ At the physical point, we define the green line by the inflection point of $\Delta$ as a function of $\mu$ for fixed $\mu_{I}$. For smaller sigma masses, the green line represents a first-order transition [35]. This line ends at a critical point.
} 




FIG. 2. Phase diagram in the $\mu-\mu_{I}$ plane at the physical point in the homogeneous case at $T=0$. See main text for details.

potential depends on $\mu$ and $\mu_{I}$, and therefore the quark and isospin densities are nonzero.

Since we have determined the parameters of the Lagrangian such that the pion propagator including the self-energy has a pole at $m_{\pi}=140 \mathrm{MeV}$, the onset of pion condensation is exactly at $\mu_{I}=\frac{1}{2} m_{\pi}$ [16]. We show this explicitly in Appendix C. The result can be understood as follows. The energy of a zero-momentum pion in the vacuum phase is $m_{\pi}-2 \mu_{I}$. If it is a second-order transition it must take place exactly at a point where the (mediumdependent) mass of the pion drops to zero because in the condensed phase there is a massless Goldstone mode associated with the breaking of the $U(1)$ symmetry. ${ }^{3}$ If one uses matching at tree level, there will be finite corrections to this relation. Likewise, if one uses the effective potential itself to define the pion mass, one uses the pion self-energy at zero external momentum and so the pole of the propagator is not at the physical mass. Again there will be finite corrections to $\mu_{I}=\frac{1}{2} m_{\pi}$ and in some cases, the deviation can be substantial [35]. Finally, we mention that the lattice result [13-15] for the onset of pion condensation at $T=0$ for $2+1$ dynamical quarks is in agreement with this. Likewise, their simulations strongly suggest that the transition is second order in the $O(2)$ universality class in agreement with expectations.

In Fig. 3, we show $\Delta$ (blue line) and $\rho$ (red line) as functions of the chemical potential for fixed value of the isospin chemical potential, $\mu_{I}=0$ in the upper panel and $\mu_{I}=90 \mathrm{MeV}$ in the lower panel. For $\mu_{I}=0$ there is no pion condensate, and there is a crossover at $\mu=323.6 \mathrm{MeV}$. For sigma masses below $600 \mathrm{MeV}$, this transition is first order.

\section{B. Inhomogeneous chiral condensate versus homogeneous pion condensate}

In this section, we generalize our result to nonzero $q$, i.e. we allow for an inhomogeneous chiral condensate.

\footnotetext{
${ }^{3}$ It is the $U_{I_{3}}(1)$ symmetry mentioned in Sec. II which is broken by the pion condensate.
}
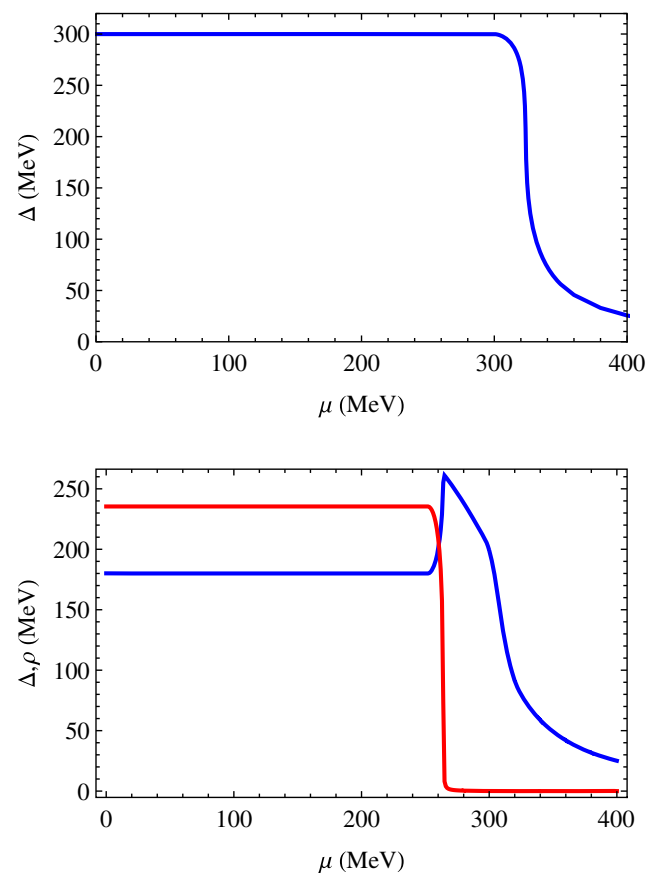

FIG. 3. Chiral condensate (blue line) and pion condensate (red line) as functions of the chemical potential $\mu$ for $\mu_{I}=0$ (upper panel) and $\mu_{I}=90 \mathrm{MeV}$ (lower panel) at the physical point and $T=0$.

It is known from earlier studies of inhomogeneous phases [41] in the NJL and QM models that the size of the region where an inhomogeneous phase exists depends rather sensitively on the mass of the pion. In Fig. 4, we show the lower and upper values of the chemical potential $\mu$ for which an inhomogeneous phase exists as a function of $m_{\pi}$ at zero isospin chemical potential. The curves meet at $m_{\pi}^{c}=37.1 \mathrm{MeV}$, beyond which no inhomogeneous phase exists. In particular, no inhomogeneous phase exists at the physical point. The mechanism behind this is as follows. The symmetry-breaking term $\sim-m_{\pi}^{2} f_{\pi}^{2} \cos (q z) \delta_{q, 0}$ is nonzero only for $q=0$. When the pion mass is sufficiently large, this term is large enough for the homogeneous phase to be preferred over the inhomogeneous one. This is in

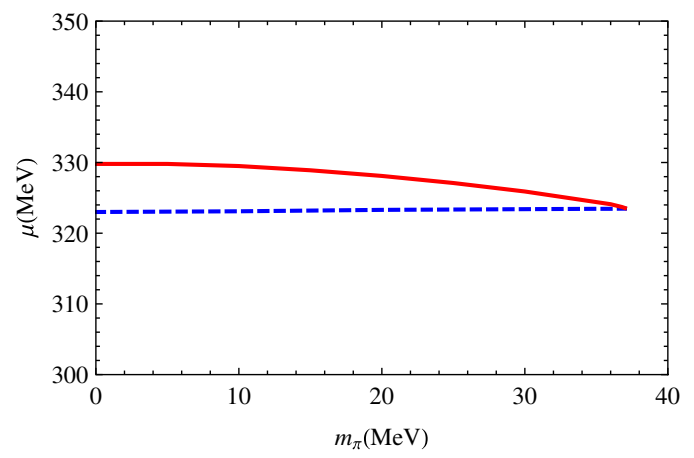

FIG. 4. Lower and upper limits of the chemical potential $\mu$ where an inhomogeneous phase exists as a function of $m_{\pi}$ for $\mu_{I}=0$. 


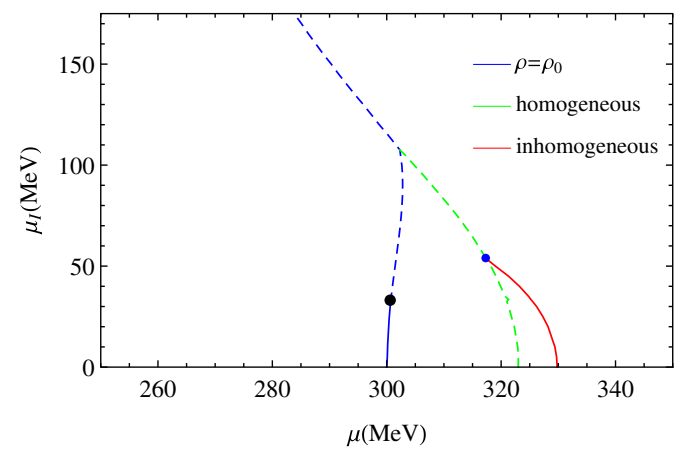

FIG. 5. Phase diagram in the $\mu-\mu_{I}$ plane at $T=0$ in the chiral limit. See main text for details.

contrast to the results of [41], where an inhomogeneous phase exists all the way up to $m_{\pi}=140 \mathrm{MeV}$. We can think of at least two reasons for this qualtitative difference. Firstly, in Ref. [41] tree-level parameters were used. Secondly, a solitonic ansatz for the inhomogenity was used, and it is possible that this can sustain a inhomogeneous phase for larger pion masses.

In Fig. 5, we show the phase diagram in the $\mu-\mu_{I}$ plane at vanishing temperature for $m_{\pi}=0$. Solid lines indicate second-order transitions, while dashed lines indicate first-order transitions. The black dot is the end point of the first-order line. To the left of the blue line there is a homogeneous pion condensate that does not change with increasing chemical potential and is equal to $\rho_{0}=$ $\rho\left(\mu=0, \mu_{I}\right)$. Between the blue and green line the pion condensate decreases and the quark density is nonzero. In both homogeneous phases we find $\Delta=0$, except for $\mu_{I}=0 .{ }^{4}$ The region between the green and the red line is the inhomogeneous phase, where the chiral condensate and wave vector $q$ are nonzero. In this phase, the pion condensate vanishes, implying that an inhomogeneous chiral condensate and a homogeneous pion condensate do not coexist. Similar conclusions have been drawn in studies of the $1+1$ dimensional NJL model $[44,45]$. Finally, the region to the right of red, blue, and green line segments is the symmetric phase, where $\Delta=\rho=q=0$. The blue dot marks the Lifshitz point where the homogeneous, inhomogeneous and chirally symmetric phases connect.

In Fig. 6, we show a cross section of the phase diagram in Fig. 5 in the chiral limit for $\mu=0$. The pion condensate is shown as a function of the isospin chemical potential $\mu_{I}$. We notice that the chiral condensate in the vacuum immediately vanishes once $\mu_{I}>0$ and is rotated to a pion condensate with the value $\rho=m_{q}$. The pion condensate increases further as we increase the isospin chemical potential $\mu_{I}$.

\footnotetext{
${ }^{4}$ On the $\mu$-axis, i.e. for $\mu_{I}=0$, the effective potential is a function of $\Delta^{2}+\rho^{2}$ with a minimum at $m_{q}^{2}$ up to $\mu=m_{q}$. Since the critical isospin chemical potential is $\mu_{I}^{c}=0$, pion condensation starts away from the $\mu$-axis, and we chose $\Delta=m_{q}$ as the minimum.
}

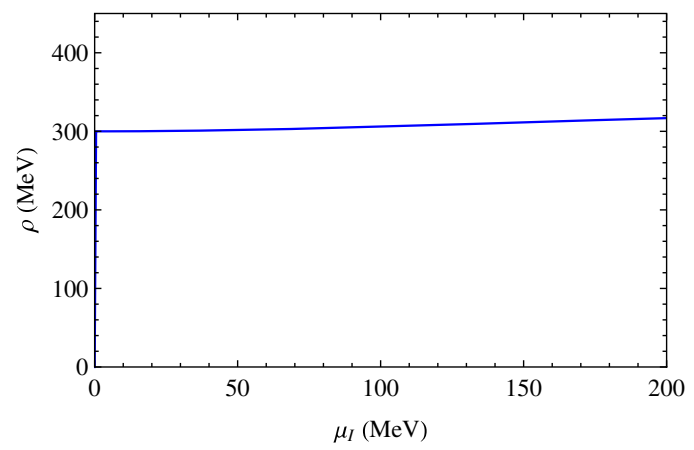

FIG. 6. Pion condensate as a function of the chemical potential $\mu_{I}$ in the chiral limit for $\mu=0$.

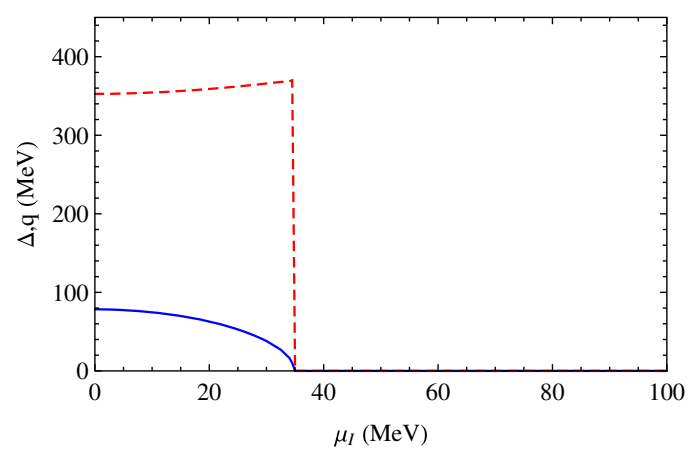

FIG. 7. Chiral condensate $\Delta$ (blue line) and wave vector $q$ (red line) as a function of the isospin chemical potential $\mu_{I}$ in the chiral limit for $\mu=325 \mathrm{MeV}$.

In Fig. 7, we show a cross section of the phase diagram in Fig. 5 in the chiral limit for $\mu=325 \mathrm{MeV}$. The chiral condensate is the blue line, and the wave vector is the red line. The transition to the symmetric phase is of second order. Note that the pion condensate is zero in the inhomogeneous phase.

In Fig. 8, we show the chiral condensate (blue line), wave vector (red line), and pion condensate (green line) as functions of the chemical potential $\mu$ in the chiral limit for $\mu_{I}=5 \mathrm{MeV}$. In the homogeneous phase, a pion condensate is favored over a chiral condensate. At the first-order transition at $\mu=323 \mathrm{MeV}$, the pion condensate drops to

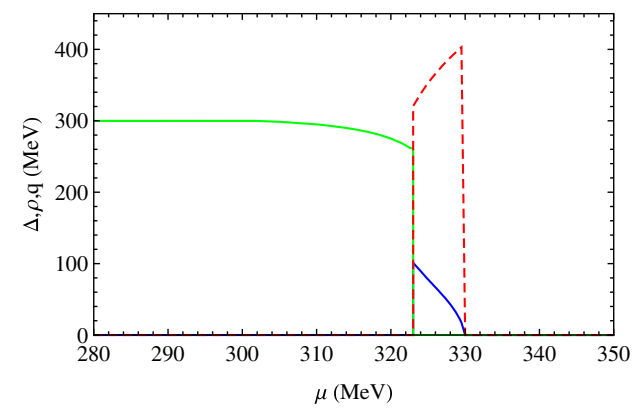

FIG. 8. Chiral condensate (blue line), wave vector (red line), and pion condensate (green line) as functions of the chemical potential $\mu$ in the chiral limit for $\mu_{I}=5 \mathrm{MeV}$. 


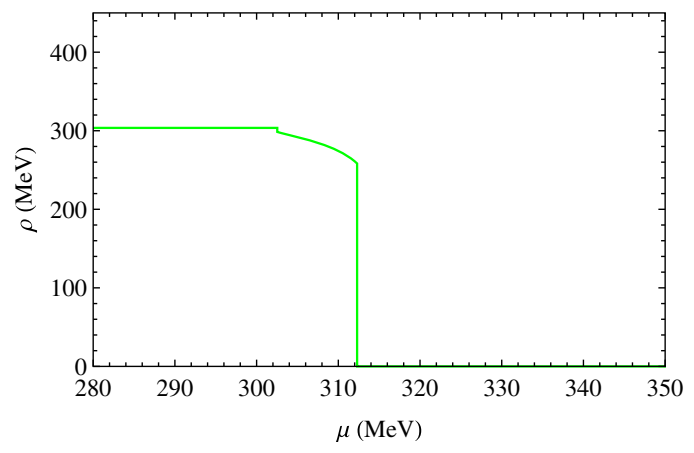

FIG. 9. Pion condensate $\rho$ as a function of the chemical potential $\mu$ in the chiral limit for $\mu_{I}=75 \mathrm{MeV}$.

zero, and we enter a phase with an inhomogeneous chiral condensate. At $\mu=329.8 \mathrm{MeV}$, there is a second-order transition to the symmetric phase.

In Fig. 9, we show the pion condensate as a function of the chemical potential in the chiral limit and for $\mu_{I}=75 \mathrm{MeV}$. There are two first-order transitions at $\mu=302.5 \mathrm{MeV}$ and $\mu=312.3 \mathrm{MeV}$, respectively, where the value of the pion condensate jumps discontinuously.

\section{SUMMARY}

In the present paper, we have studied the phases of QCD at $T=0$ in the $\mu-\mu_{I}$ plane using the quark-meson model as a low-energy effective model. Combining the $\overline{\mathrm{MS}}$ and OS schemes, we have determined the parameters of the model, whose values are consistent with the approximation that we used for the effective potential. In contrast to other model calculations, where the parameters are fixed at tree level, our method guarantees that the critical isospin chemical potential at $T=0$ is exactly at $\mu_{I}=\frac{1}{2} m_{\pi}$.

Moreover, we found that the existence of an inhomogeneous chiral condensate depends on the value of the pion mass, which is in agreement with earlier model calculations [41]. Specifically, we found that the chiral density wave is disfavored for pion masses larger than approximately $37 \mathrm{MeV}$. The existence of such a critical pion mass is in contrast to the results of Ref. [41], where an inhomogeneous chiral condensate exists all the way up to $m_{\pi}=140 \mathrm{MeV}$. The difference is probably due to treelevel versus one-loop matching of the parameters as well as different ansätze for the inhomogeneity.

Finally, we mapped out the phase diagram at $T=0$ in the $\mu-\mu_{I}$ plane both in the chiral limit and at the physical point. In the chiral limit, we have seen that there is a region where an inhomogeneous chiral condensate is favored. The corresponding Lifshitz point is a tricritical point, as three phases meet. At the physical point, the phase diagram is in qualitative agreement with the mean-field calculations in [35]. However, they have also performed a functional renormalization group (FRG) calculation of the phase diagram and including the mesonic fluctuations gives rise to qualitative differences. One important difference is that the transition to a Bose-condensed phase is second order in the entire $\mu-\mu_{I}$ plane. It would be very interesting to perform an FRG calculation including inhomogeneous phases to see whether the mean-field picture found here will change.

\section{ACKNOWLEDGMENTS}

The authors would like to thank Tomas Brauner for useful discussions. P.K. would like to thank the Department of Physics at NTNU for kind hospitality during the latter stages of this work.

\section{APPENDIX A: INTEGRALS}

With dimensional regularization, the momentum integral is generalized to $d=3-2 \epsilon$ spatial dimensions. We define the dimensionally regularized integral by

$$
\int_{p}=\left(\frac{e^{\gamma_{E}} \Lambda^{2}}{4 \pi}\right)^{\epsilon} \int \frac{d^{d} p}{(2 \pi)^{d}}
$$

where $\Lambda$ is the renormalization scale in the modified minimal subtraction scheme $\overline{\mathrm{MS}}$. It is convenient to write the integral as

$$
\int_{p}=\left(\frac{e^{\gamma_{E}} \Lambda^{2}}{4 \pi}\right)^{\epsilon} \int \frac{d^{d-1} p_{\perp}}{(2 \pi)^{d-1}} \int_{-\infty}^{\infty} \frac{d p_{\|}}{2 \pi} .
$$

In order to calculate the effective potential, we need the vacuum integrals

$$
\begin{gathered}
\int_{p} \sqrt{p^{2}+M^{2}}=-\frac{M^{4}}{(4 \pi)^{2}}\left(\frac{e^{\gamma_{E}} \Lambda^{2}}{M^{2}}\right)^{\epsilon} \Gamma(-2+\epsilon)=-\frac{M^{4}}{2(4 \pi)^{2}}\left(\frac{\Lambda^{2}}{M^{2}}\right)^{\epsilon}\left[\frac{1}{\epsilon}+\frac{3}{2}+\mathcal{O}(\epsilon)\right], \\
\int_{p} \frac{1}{\sqrt{p^{2}+M^{2}}}=-\frac{2 M^{2}}{(4 \pi)^{2}}\left(\frac{e^{\gamma_{E}} \Lambda^{2}}{M^{2}}\right)^{\epsilon} \Gamma(-1+\epsilon)=-\frac{2 M^{2}}{(4 \pi)^{2}}\left(\frac{\Lambda^{2}}{M^{2}}\right)^{\epsilon}\left[\frac{1}{\epsilon}+1+\mathcal{O}(\epsilon)\right], \\
\int_{p} \frac{1}{\left(p^{2}+M^{2}\right)^{\frac{3}{2}}}=\frac{4}{(4 \pi)^{2}}\left(\frac{e^{\gamma_{E}} \Lambda^{2}}{M^{2}}\right)^{\epsilon} \Gamma(\epsilon)=\frac{4}{(4 \pi)^{2}}\left(\frac{\Lambda^{2}}{M^{2}}\right)^{\epsilon}\left[\frac{1}{\epsilon}+\mathcal{O}(\epsilon)\right], \\
\int_{p} \frac{\left(p_{\perp}^{2}+\rho^{2}\right)}{\left(p^{2}+\Delta^{2}+\rho^{2}\right)^{\frac{3}{2}}}=-\frac{4 \Delta^{2}}{(4 \pi)^{2}}\left(\frac{e^{\gamma_{E}} \Lambda^{2}}{\Delta^{2}+\rho^{2}}\right)^{\epsilon} \Gamma(\epsilon)=-\frac{4 \Delta^{2}}{(4 \pi)^{2}}\left(\frac{\Lambda^{2}}{\Delta^{2}+\rho^{2}}\right)^{\epsilon}\left[\frac{1}{\epsilon}+\mathcal{O}(\epsilon)\right]
\end{gathered}
$$




$$
\begin{aligned}
\int_{p} \frac{\left(p_{\perp}^{2}+\rho^{2}\right)\left(4 \Delta^{2}-\rho^{2}+4 p_{\|}^{2}-p_{\perp}^{2}\right)}{\left(p_{\perp}^{2}+p_{\|}^{2}+M^{2}\right)^{\frac{7}{2}}} & =\frac{16}{3(4 \pi)^{2}}\left(\frac{e^{\gamma_{E}} \Lambda^{2}}{\Delta^{2}+\rho^{2}}\right)^{\epsilon} \frac{\Delta^{2}}{\left(\Delta^{2}+\rho^{2}\right)^{2}}\left[(1-\epsilon) \Delta^{2}+2 \rho^{2}\right] \Gamma(1+\epsilon) \\
& =\frac{16}{3(4 \pi)^{2}} \frac{\Delta^{2}\left(\Delta^{2}+2 \rho^{2}\right)}{\left(\Delta^{2}+\rho^{2}\right)^{2}}[1+\mathcal{O}(\epsilon)], \\
\int_{p} \frac{\left(4 \Delta^{2}-\rho^{2}+4 p_{\|}^{2}-p_{\perp}^{2}\right)}{\left(p^{2}+\Delta^{2}+\rho^{2}\right)^{\frac{7}{2}}} & =\frac{16}{3(4 \pi)^{2}}\left(\frac{e^{\gamma_{E}} \Lambda^{2}}{\Delta^{2}+\rho^{2}}\right)^{\epsilon} \frac{\Delta^{2}}{\left(\Delta^{2}+\rho^{2}\right)^{2}} \Gamma(2+\epsilon)=\frac{16}{3(4 \pi)^{2}} \frac{\Delta^{2}}{\left(\Delta^{2}+\rho^{2}\right)^{2}}[1+\mathcal{O}(\epsilon)] .
\end{aligned}
$$

\section{APPENDIX B: PARAMETER FIXING}

In this appendix, we briefly discuss the fixing of the model parameters. At tree level, the relations between these parameters and the physical quantities are given by Eqs. (15)-(16). In the on shell scheme, the divergent loop integrals are regularized using dimensional regularization, but the counterterms are defined differently from those in the minimal subtraction scheme. The counterterms in the on shell scheme are chosen so that they exactly cancel the loop corrections to the self-energies ${ }^{5}$ and couplings evaluated on the mass shell, and consequently, the renormalized parameters are independent of the renormalization scale and satisfy the tree-level relations [50-52]. In the $\overline{\mathrm{MS}}$ scheme, the counterterms are chosen so that they cancel only the poles in $\epsilon$ of the loop corrections. The bare parameters are the same in the two schemes and so we can relate the corresponding renormalized parameters. The running parameters in the $\overline{\mathrm{MS}}$ scheme can therefore be expressed in terms of the physical masses $m_{\sigma}, m_{\pi}$, and $m_{q}$ as well the pion decay constant. In Ref. [48] we found

$$
\begin{aligned}
m_{\overline{\mathrm{MS}}}^{2}= & m^{2}+8 i g^{2} N_{c}\left[A\left(m_{q}^{2}\right)+\frac{1}{4}\left(m_{\sigma}^{2}-4 m_{q}^{2}\right) B\left(m_{\sigma}^{2}\right)-\frac{3}{4} m_{\pi}^{2} B\left(m_{\pi}^{2}\right)\right]-\delta m_{\overline{\mathrm{MS}}}^{2} \\
= & m^{2}+\frac{4 g^{2} N_{c}}{(4 \pi)^{2}}\left[m^{2} \log \frac{\Lambda^{2}}{m_{q}^{2}}-2 m_{q}^{2}-\frac{1}{2}\left(m_{\sigma}^{2}-4 m_{q}^{2}\right) F\left(m_{\sigma}^{2}\right)+\frac{3}{2} m_{\pi}^{2} F\left(m_{\pi}^{2}\right)\right], \\
\lambda_{\overline{\mathrm{MS}}}= & \lambda-\frac{12 i g^{2} N_{c}}{f_{\pi}^{2}}\left(m_{\sigma}^{2}-4 m_{q}^{2}\right) B\left(m_{\sigma}^{2}\right)+\frac{12 i g^{2} N_{c}}{f_{\pi}^{2}} m_{\pi}^{2} B\left(m_{\pi}^{2}\right)-4 i \lambda g^{2} N_{c}\left[B\left(m_{\pi}^{2}\right)+m_{\pi}^{2} B^{\prime}\left(m_{\pi}^{2}\right)\right]-\delta \lambda_{\overline{\mathrm{MS}}} \\
= & \lambda+\left\{\frac { 1 2 g ^ { 2 } N _ { c } } { ( 4 \pi ) ^ { 2 } f _ { \pi } ^ { 2 } } \left[\left(m_{\sigma}^{2}-4 m_{q}^{2}\right)\left(\log \frac{\Lambda^{2}}{m_{q}^{2}}+F\left(m_{\sigma}^{2}\right)\right)+m_{\sigma}^{2}\left(\log \frac{\Lambda^{2}}{m_{q}^{2}}+F\left(m_{\pi}^{2}\right)+m_{\pi}^{2} F^{\prime}\left(m_{\pi}^{2}\right)\right)\right.\right. \\
& \left.\left.-m_{\pi}^{2}\left(2 \log \frac{\Lambda^{2}}{m_{q}^{2}}+2 F\left(m_{\pi}^{2}\right)+F^{\prime}\left(m_{\pi}^{2}\right)\right)\right]\right\}, \\
g_{\overline{\mathrm{MS}}}^{2}= & g^{2}-4 i g^{4} N_{c}\left[B\left(m_{\pi}^{2}\right)+m_{\pi}^{2} B^{\prime}\left(m_{\pi}^{2}\right)\right]-\delta g_{\overline{\mathrm{MS}}}^{2}=\frac{m_{q}^{2}}{f_{\pi}^{2}}\left\{1+\frac{4 g^{2} N_{c}}{(4 \pi)^{2}}\left[\log \frac{\Lambda^{2}}{m_{q}^{2}}+F\left(m_{\pi}^{2}\right)+m_{\pi}^{2} F^{\prime}\left(m_{\pi}^{2}\right)\right]\right\}, \\
h_{\overline{\mathrm{MS}}}= & h-2 i g^{2} N_{c} m_{\pi}^{2} f_{\pi}\left[B\left(m_{\pi}^{2}\right)-m_{\pi}^{2} B^{\prime}\left(m_{\pi}^{2}\right)\right]-\delta h_{\overline{\mathrm{MS}}}=h\left\{1+\frac{2 g^{2} N_{c}}{(4 \pi)^{2}}\left[\log \frac{\Lambda^{2}}{m_{q}^{2}}+F\left(m_{\pi}^{2}\right)-m_{\pi}^{2} F^{\prime}\left(m_{\pi}^{2}\right)\right]\right\},
\end{aligned}
$$

where $A\left(m_{q}^{2}\right), B\left(p^{2}\right)$, and $B^{\prime}\left(p^{2}\right)$ are integrals in $d=4-2 \epsilon$ dimensions in Minkowski space. Going to Euclidean space, they can be straightforwardly computed and read

$$
A\left(m_{q}^{2}\right)=\int_{p} \frac{1}{p^{2}-m_{q}^{2}}=\frac{i m_{q}^{2}}{(4 \pi)^{2}}\left(\frac{\Lambda^{2}}{m_{q}^{2}}\right)\left[\frac{1}{\epsilon}+1+\mathcal{O}(\epsilon)\right],
$$

\footnotetext{
${ }^{5}$ And such that the residues of the propagators evaluated on the mass shell are unity.
} 


$$
\begin{aligned}
B\left(p^{2}\right) & =\int_{p} \frac{1}{\left(k^{2}-m_{q}^{2}\right)\left[(k+p)^{2}-m_{q}^{2}\right]} \\
& =\frac{i}{(4 \pi)^{2}}\left(\frac{\Lambda^{2}}{m_{q}^{2}}\right)\left[\frac{1}{\epsilon}+F\left(p^{2}\right)+\mathcal{O}(\epsilon)\right], \\
B^{\prime}\left(p^{2}\right) & =\frac{i}{(4 \pi)^{2}} F^{\prime}\left(p^{2}\right)
\end{aligned}
$$

Here we have defined

$$
\begin{aligned}
F\left(p^{2}\right) & =2-2 r \arctan \left(\frac{1}{r}\right), \\
F^{\prime}\left(p^{2}\right) & =\frac{4 m_{q}^{2} r}{p^{2}\left(4 m_{q}^{2}-r^{2}\right)} \arctan \left(\frac{1}{r}\right)-\frac{1}{p^{2}},
\end{aligned}
$$

with $r=\sqrt{\frac{4 m_{q}^{2}}{p^{2}}-1}$.

The running parameters satisfy the following renormalization group equations:

$$
\begin{aligned}
\Lambda \frac{d m_{\overline{\mathrm{MS}}}^{2}(\Lambda)}{d \Lambda} & =\frac{8 N_{c} m_{\overline{\mathrm{MS}}}^{2}(\Lambda) g_{\overline{\mathrm{MS}}}^{2}(\Lambda)}{(4 \pi)^{2}}, \\
\Lambda \frac{d g_{\overline{\mathrm{MS}}}^{2}(\Lambda)}{d \Lambda} & =\frac{8 N_{c} g_{\overline{\mathrm{MS}}}^{4}(\Lambda)}{(4 \pi)^{2}} \\
\Lambda \frac{d \lambda_{\overline{\mathrm{MS}}}(\Lambda)}{d \Lambda} & =\frac{16 N_{c}}{(4 \pi)^{2}}\left[\lambda \overline{\mathrm{MS}}(\Lambda) g_{\overline{\mathrm{MS}}}^{2}(\Lambda)-6 g_{\overline{\mathrm{MS}}}^{4}(\Lambda)\right], \\
\Lambda \frac{d h_{\overline{\mathrm{MS}}}(\Lambda)}{d \Lambda} & =\frac{4 N_{c} g_{\overline{\mathrm{MS}}}^{2}(\Lambda) h_{\overline{\mathrm{MS}}}(\Lambda)}{(4 \pi)^{2}} .
\end{aligned}
$$

The solutions to Eqs. (B10)-(B13) are

$$
\begin{aligned}
& m_{\overline{\mathrm{MS}}}^{2}(\Lambda)=\frac{m_{0}^{2}}{1-\frac{4 g_{0}^{2} N_{c}}{(4 \pi)^{2}} \log \frac{\Lambda^{2}}{\Lambda_{0}^{2}}} . \\
& g_{\overline{\mathrm{MS}}}^{2}(\Lambda)=\frac{g_{0}^{2}}{1-\frac{4 g_{0}^{2} N_{c}}{(4 \pi)^{2}} \log \frac{\Lambda^{2}}{\Lambda_{0}^{2}}}, \\
& \lambda_{\overline{\mathrm{MS}}}(\Lambda)=\frac{\lambda_{0}-\frac{48 g_{0}^{4} N_{c}}{(4 \pi)^{2}} \log \frac{\Lambda^{2}}{\Lambda_{0}^{2}}}{\left(1-\frac{4 g_{0}^{2} N_{c}}{(4 \pi)^{2}} \log \frac{\Lambda^{2}}{\Lambda_{0}^{2}}\right)^{2}},
\end{aligned}
$$

$$
h_{\overline{\mathrm{MS}}}(\Lambda)=\frac{h_{0}}{1-\frac{2 g_{0}^{2} N_{c}}{(4 \pi)^{2}} \log \frac{\Lambda^{2}}{\Lambda_{0}^{2}}},
$$

where $m_{0}^{2}, g_{0}^{2}, \lambda_{0}$ and $h_{0}$, are the values of the running parameters at the scale $\Lambda_{0}$. We choose $\Lambda_{0}$ to satisfy

$$
\log \frac{\Lambda_{0}^{2}}{m_{q}^{2}}+F\left(m_{\pi}^{2}\right)+m_{\pi}^{2} F^{\prime}\left(m_{\pi}^{2}\right)=0 .
$$

$F\left(m_{\pi}^{2}\right)$ and $m_{\pi}^{2} F^{\prime}\left(m_{\pi}^{2}\right)$ vanish in the chiral limit which implies that $\Lambda_{0}=m_{q}$. We can now evaluate Eqs. (B1)-(B4) at $\Lambda=\Lambda_{0}$ to find $m_{0}^{2}, \lambda_{0}, g_{0}^{2}$, and $h_{0}$. Inserting Eqs. (B14)-(B17) into Eq. (28) using the results for $m_{0}^{2}$, $\lambda_{0}, g_{0}^{2}$, and $h_{0}$, we obtain the final result Eq. (29).

\section{APPENDIX C: ONSET OF PION CONDENSATION}

In order to show that the onset of pion condensation is exactly at $\mu_{I}=\frac{1}{2} m_{\pi}$, we expand the one-loop contribution to the free energy in powers of $\rho$ to fourth order to obtain an effective Ginzburg-Landau energy functional. For simplicity, we consider the case $\mu=0$ where the finite-density contribution (31) vanishes.

After renormalization, the one-loop effective potential will be of the form

$$
V_{1-\text { loop }}=\alpha_{0}+\alpha_{2} \rho^{2}+\alpha_{4} \rho^{4},
$$

where the coefficients $\alpha_{i}$ depend on the physical quantities $m_{\sigma}, m_{\pi}, f_{\pi}, \mu_{I}$ and $\Delta$. The critical isospin chemical potential $\mu_{I}^{c}$ is defined by $\alpha_{2}=0$ evaluated at $\Delta=m_{q}$, i.e. its vacuum value. If $\alpha_{4}>0$ at $\mu_{I}^{c}$ and $\Delta=m_{q}$, then there is a second order transition at $\mu_{I}^{c}$.

Setting $q=0$ in Eq. (23) the one-loop vacuum energy reduces to

$$
V_{1}=-2 N_{c} \int_{p} \sqrt{\left(\sqrt{p^{2}+\Delta^{2}} \pm \mu_{I}\right)^{2}+\rho^{2}} .
$$

Expanding Eq. (C2) in powers of $\rho$ up to fourth order, we find

$$
V_{1}=-2 N_{c} \int_{p}\left[\sqrt{p^{2}+\Delta^{2}} \pm \mu_{I}+\frac{1}{2} \frac{\rho^{2}}{\sqrt{p^{2}+\Delta^{2}} \pm \mu_{I}}-\frac{1}{8} \frac{\rho^{4}}{\left(\sqrt{p^{2}+\Delta^{2}} \pm \mu_{I}\right)^{3}}\right] .
$$

In order to isolate the divergence, we expand the different terms in Eq. (C3) in powers of $\mu_{I}$ to the appropriate power order, then add and subtract suitable terms. This yields

$$
V_{1}=-4 N_{c} \int_{p}\left[\sqrt{p^{2}+\Delta^{2}}+\frac{1}{2} \rho^{2}\left(\frac{1}{\sqrt{p^{2}+\Delta^{2}}}+\frac{\mu_{I}^{2}}{\left(p^{2}+\Delta^{2}\right)^{\frac{3}{2}}}\right)-\frac{1}{8} \frac{\rho^{4}}{\left(p^{2}+\Delta^{2}\right)^{\frac{3}{2}}}\right]+V_{\mathrm{fin}, 1}+V_{\mathrm{fin}, 2},
$$

where the finite terms can be calculated directly in $d=3$ and read 


$$
\begin{aligned}
& V_{\mathrm{fin}, 1}=-2 N_{c} \rho^{2} \int_{p}\left[\frac{\sqrt{p^{2}+\Delta^{2}}}{p^{2}+\Delta^{2}-\mu_{I}^{2}}-\frac{1}{\sqrt{p^{2}+\Delta^{2}}}-\frac{\mu_{I}^{2}}{\left(p^{2}+\Delta^{2}\right)^{\frac{3}{2}}}\right]=-\frac{16 N_{c}}{(4 \pi)^{2}} \rho^{2} \mu_{I}^{2}\left[1-s \arctan \left(\frac{1}{s}\right)\right], \\
& V_{\mathrm{fin}, 2}=\frac{1}{4} N_{c} \rho^{4} \int_{p}\left[\frac{1}{\left(\sqrt{p^{2}+\Delta^{2}} \pm \mu_{I}\right)^{3}}-\frac{2}{\left(p^{2}+\Delta^{2}\right)^{\frac{3}{2}}}\right]=\frac{2 N_{c}}{(4 \pi)^{2}} \rho^{4}\left[\frac{1}{s^{2}}+\frac{1}{s^{3}}\left(\frac{3 \Delta^{2}}{\mu_{I}^{2}}-2\right) \arctan \left(\frac{1}{s}\right)\right],
\end{aligned}
$$

where $s=\sqrt{\frac{\Delta^{2}}{\mu_{I}^{2}}-1}$. In analogy with Eq. (28), we obtain the renormalized vacuum potential through order $\rho^{4}$

$$
\begin{aligned}
V_{1-\text { loop }}= & \frac{1}{2} \frac{m_{\overline{\mathrm{MS}}}^{2}}{g_{\overline{\mathrm{MS}}}^{2}} \Delta^{2}+\frac{1}{2} \frac{m_{\overline{\mathrm{MS}}}^{2}-4 \mu_{I}^{2}}{g_{\overline{\mathrm{MS}}}^{2}} \rho^{2}+\frac{\lambda \overline{\overline{\mathrm{MS}}}}{24 g_{\overline{\mathrm{MS}}}^{4}}\left(\Delta^{2}+\rho^{2}\right)^{2}-\frac{h_{\overline{\mathrm{MS}}}}{g_{\overline{\mathrm{MS}}}} \Delta \cos (q z) \delta_{q, 0} \\
& +\frac{2 N_{c}}{(4 \pi)^{2}}\left\{\left(\Delta^{2}+\rho^{2}\right)^{2} \log \frac{\Lambda^{2}}{\Delta^{2}}+4 \rho^{2} \mu_{I}^{2} \log \frac{\Lambda^{2}}{\Delta^{2}}+\frac{3}{2} \Delta^{4}+2 \Delta^{2} \rho^{2}\right\}+V_{\text {fin }, 1}+V_{\text {fin }, 2} .
\end{aligned}
$$

Using Eqs. (A3)-(A5) as well as (B14)-(B17), the renormalized effective potential up to order $\rho^{4}$ is

$$
\begin{aligned}
V_{1 \text {-loop }}= & \frac{3}{4} m_{\pi}^{2} f_{\pi}^{2}\left\{1-\frac{4 m_{q}^{2} N_{c}}{(4 \pi)^{2} f_{\pi}^{2}} m_{\pi}^{2} F^{\prime}\left(m_{\pi}^{2}\right)\right\} \frac{\Delta^{2}+\rho^{2}}{m_{q}^{2}} \\
& -\frac{1}{4} m_{\sigma}^{2} f_{\pi}^{2}\left\{1+\frac{4 m_{q}^{2} N_{c}}{(4 \pi)^{2} f_{\pi}^{2}}\left[\left(1-\frac{4 m_{q}^{2}}{m_{\sigma}^{2}}\right) F\left(m_{\sigma}^{2}\right)+\frac{4 m_{q}^{2}}{m_{\sigma}^{2}}-F\left(m_{\pi}^{2}\right)-m_{\pi}^{2} F^{\prime}\left(m_{\pi}^{2}\right)\right]\right\} \frac{\Delta^{2}+\rho^{2}}{m_{q}^{2}} \\
& -2 \mu_{I}^{2} f_{\pi}^{2}\left\{1-\frac{4 m_{q}^{2} N_{c}}{(4 \pi)^{2} f_{\pi}^{2}}\left[\log \frac{\Delta^{2}}{m_{q}^{2}}+F\left(m_{\pi}^{2}\right)+m_{\pi}^{2} F^{\prime}\left(m_{\pi}^{2}\right)-2+2 s \arctan \frac{1}{s}\right]\right\} \frac{\rho^{2}}{m_{q}^{2}} \\
& +\frac{1}{8} m_{\sigma}^{2} f_{\pi}^{2}\left\{1-\frac{4 m_{q}^{2} N_{c}}{(4 \pi)^{2} f_{\pi}^{2}}\left[\frac{4 m_{q}^{2}}{m_{\sigma}^{2}} \log \frac{\Delta^{2}}{m_{q}^{2}}-\left(1-\frac{4 m_{q}^{2}}{m_{\sigma}^{2}}\right) F\left(m_{\sigma}^{2}\right)+F\left(m_{\pi}^{2}\right)+m_{\pi}^{2} F^{\prime}\left(m_{\pi}^{2}\right)\right]\right\} \frac{\left(\Delta^{2}+\rho^{2}\right)^{2}}{m_{q}^{4}} \\
& -\frac{1}{8} m_{\pi}^{2} f_{\pi}^{2}\left[1-\frac{4 m_{q}^{2} N_{c}}{(4 \pi)^{2} f_{\pi}^{2}} m_{\pi}^{2} F^{\prime}\left(m_{\pi}^{2}\right)\right] \frac{\left(\Delta^{2}+\rho^{2}\right)^{2}}{m_{q}^{4}}-m_{\pi}^{2} f_{\pi}^{2}\left[1-\frac{4 m_{q}^{2} N_{c}}{(4 \pi)^{2} f_{\pi}^{2}} m_{\pi}^{2} F^{\prime}\left(m_{\pi}^{2}\right)\right] \frac{\Delta}{m_{q}} \\
& +\frac{2 N_{c}}{(4 \pi)^{2}}\left\{\frac{3}{2} \Delta^{4}+2 \Delta^{2} \rho^{2}+\left[\frac{1}{s^{2}}+\frac{1}{s^{3}}\left(\frac{3 \Delta^{2}}{\mu_{I}^{2}}-2\right) \arctan \left(\frac{1}{s}\right)\right] \rho^{4}\right\} .
\end{aligned}
$$

From the effective potential Eq. (C8), we can read off the coefficients $\alpha_{2}$ and $\alpha_{4}$. Evaluated at $\Delta=m_{q}$, we find

$$
\begin{aligned}
\alpha_{2}= & \frac{1}{2} \frac{f_{\pi}^{2}}{m_{q}^{2}}\left\{m_{\pi}^{2}\left[1-\frac{4 m_{q}^{2} N_{c}}{(4 \pi)^{2} f_{\pi}^{2}} m_{\pi}^{2} F^{\prime}\left(m_{\pi}^{2}\right)\right]-4 \mu_{I}^{2}\left[1-\frac{4 m_{q}^{2} N_{c}}{(4 \pi)^{2} f_{\pi}^{2}}\left(F\left(m_{\pi}^{2}\right)+m_{\pi}^{2} F^{\prime}\left(m_{\pi}^{2}\right)-F\left(4 \mu_{I}^{2}\right)\right)\right]\right\}, \\
\alpha_{4}= & \frac{1}{8} \frac{m_{\sigma}^{2} f_{\pi}^{2}}{m_{q}^{4}}\left\{1-\frac{4 m_{q}^{2} N_{c}}{(4 \pi)^{2} f_{\pi}^{2}}\left[-\left(1-\frac{4 m_{q}^{2}}{m_{\sigma}^{2}}\right) F\left(m_{\sigma}^{2}\right)+F\left(m_{\pi}^{2}\right)+m_{\pi}^{2} F^{\prime}\left(m_{\pi}^{2}\right)\right]\right\}-\frac{1}{8} \frac{m_{\pi}^{2} f_{\pi}^{2}}{m_{q}^{4}}\left[1-\frac{4 m_{q}^{2} N_{c}}{(4 \pi)^{2} f_{\pi}^{2}} m_{\pi}^{2} F^{\prime}\left(m_{\pi}^{2}\right)\right] \\
& +\frac{2 N_{c}}{(4 \pi)^{2}}\left[\frac{1}{s^{2}}+\frac{1}{s^{3}}\left(\frac{3 \Delta^{2}}{\mu_{I}^{2}}-2\right) \arctan \left(\frac{1}{s}\right)\right] .
\end{aligned}
$$

Thus $\alpha_{2}=0$ for $\mu_{I}^{c}=\frac{1}{2} m_{\pi}$, and we find $\alpha_{4}>0$ for all values of $m_{\sigma}$, specifically $\alpha_{4}=0.0514$ for $m_{\sigma}=600 \mathrm{MeV}$ and $m_{\pi}=140 \mathrm{MeV}$. We therefore conclude that the transition from the vacuum to the pion-condensed phase is second order and the onset is for $\mu_{I}^{c}=\frac{1}{2} m_{\pi}$. This results holds for all $\mu<m_{q}-\frac{1}{2} m_{\pi}$ when the finite-density part of the potential becomes nonzero, as seen in Fig. 2. 
[1] K. Rajagopal and F. Wilczek, At the Frontier of Particle Physics, (World Scientific, Singapore, 2001), Vol. 3, p. 2061.

[2] M. G. Alford, A. Schmitt, K. Rajagopal, and T. Schäfer, Rev. Mod. Phys. 80, 1455 (2008).

[3] K. Fukushima and T. Hatsuda, Rep. Prog. Phys. 74, 014001 (2011).

[4] E. J. Ferrer, V. de la Incera, and C. Manuel, Phys. Rev. Lett. 95, 152002 (2005); Nucl. Phys. B747, 88 (2006).

[5] K. Fukushima and H. J. Warringa, Phys. Rev. Lett. 100, 032007 (2008).

[6] J. L. Noronha and I. A. Shovkovy, Phys. Rev. D 76, 105030 (2007); 86, 049901 (2012).

[7] T. Kojo, Y. Hidaka, L. McLerran, and R. D. Pisarski, Nucl. Phys. A843, 37 (2010); A875, 94 (2012).

[8] T. Kojo, R. D. Pisarski, and A. M. Tsvelik, Phys. Rev. D 82, 074015 (2010).

[9] R. Anglani, R. Casalbuoni, M. Ciminale, N. Ippolito, R. Gatto, M. Mannarelli, and M. Ruggieri, Rev. Mod. Phys. 86, 509 (2014).

[10] M. Buballa and S. Carignano, Prog. Part. Nucl. Phys. 81, 39 (2015).

[11] J. B. Kogut and D. K. Sinclair, Phys. Rev. D 66, 014508 (2002).

[12] J. B. Kogut and D. K. Sinclair, Phys. Rev. D 66, 034505 (2002).

[13] B. B. Brandt and G. Endrodi, Proc. Sci., LATTICE2016 (2016) 039.

[14] B. B. Brandt, G. Endrodi, and S. Schmalzbauer, arXiv: 1709.10487.

[15] B. B. Brandt, G. Endrodi, and S. Schmalzbauer, arXiv: 1712.08190 [Phys. Rev. D (to be published)].

[16] D. T. Son and M. A. Stephanov, Phys. Rev. Lett. 86, 592 (2001).

[17] K. Splittorff, D. T. Son, and M. A. Stephanov, Phys. Rev. D 64, 016003 (2001).

[18] M. Loewe and C. Villavicencio, Phys. Rev. D 67, 074034 (2003).

[19] E. S. Fraga, L. F. Palhares, and C. Villavicencio, Phys. Rev. D 79, 014021 (2009).

[20] S. Carignano, L. Lepori, A. Mammarella, M. Mannarelli, and G. Pagliaroli, Eur. Phys. J. A 53, 35 (2017).

[21] D. Toublan and J. B. Kogut, Phys. Lett. B 605, 129 (2005).

[22] B. Klein, D. Toublan, and J. J. M. Verbaarschot, Phys. Rev. D 68, 014009 (2003).

[23] M. Frank, M. Buballa, and M. Oertel, Phys. Lett. B 562, 221 (2003).

[24] D. Toublan and J. B. Kogut, Phys. Lett. B 564, 212 (2003).
[25] A. Barducci, R. Casalbuoni, G. Pettini, and L. Ravagli, Phys. Rev. D 69, 096004 (2004).

[26] L. He and P.-F. Zhuang, Phys. Lett. B 615, 93 (2005).

[27] L. He, M. Jin, and P.-F. Zhuang, Phys. Rev. D 71, 116001 (2005).

[28] L. He, M. Jin, and P.-F. Zhuang, Phys. Rev. D 74, 036005 (2006).

[29] D. Ebert and K. G. Klimenko, J. Phys. G 32, 599 (2006).

[30] D. Ebert and K. G. Klimenko, Eur. Phys. J. C 46, 771 (2006).

[31] J. O. Andersen and L. Kyllingstad, J. Phys. G 37, 015003 (2010).

[32] H. Abuki, R. Anglani, R. Gatto, M. Pellicoro, and M. Ruggieri, Phys. Rev. D 79, 034032 (2009).

[33] C.-F. Mu, L. He, and Y. Liu, Phys. Rev. D 82, 056006 (2010).

[34] T. Xia, L. He, and P. Zhuang, Phys. Rev. D 88, 056013 (2013).

[35] K. Kamikado, N. Strodthoff, L. von Smekal, and J. Wambach, Phys. Lett. B 718, 1044 (2013).

[36] H. Ueda, T. Z. Nakano, A. Ohnishi, M. Ruggieri, and K. Sumiyoshi, Phys. Rev. D 88, 074006 (2013).

[37] R. Stiele, E. S. Fraga, and J. Schaffner-Bielich, Phys. Lett. B 729, 72 (2014).

[38] T. Graf, J. Schaffner-Bielich, and E. S. Fraga, Phys. Rev. D 93, 085030 (2016).

[39] J. O. Andersen, N. Haque, M. G. Mustafa, and M. Strickland, Phys. Rev. D 93, 054045 (2016).

[40] E. Nakano and T. Tatsumi, Phys. Rev. D 71, 114006 (2005).

[41] D. Nickel, Phys. Rev. D 80, 074025 (2009); Phys. Rev. Lett. 103, 072301 (2009).

[42] S. Carignano, D. Nickel, and M. Buballa, Phys. Rev. D 82, 054009 (2010).

[43] S. Carignano, M. Buballa, and B.-J. Schaefer, Phys. Rev. D 90, 014033 (2014).

[44] N. V. Gubina, K. G. Klimenko, S. G. Kurbanov, and V. Ch. Zhukovsky, Phys. Rev. D 86, 085011 (2012).

[45] P. Adhikari and J. O. Andersen, Phys. Rev. D 95, 054020 (2017).

[46] T. G. Khunjua, K. G. Klimenko, R. N. Zhokhov, and V. C. Zhukovsky, Phys. Rev. D 95, 105010 (2017).

[47] F. Dautry and E. M. Nyman, Nucl. Phys. A319, 323 (1979).

[48] P. Adhikari, J. O. Andersen, and P. Kneschke, Phys. Rev. D 96, 016013 (2017).

[49] T. D. Cohen, Phys. Rev. Lett. 91, 222001 (2003).

[50] A. Sirlin, Phys. Rev. D 22, 971 (1980).

[51] A. Sirlin, Phys. Rev. D 29, 89 (1984).

[52] M. Bohm, H. Spiesberger, and W. Hollik, Fortschr. Phys. 34, 687 (1986). 